Ann. Biol. onim. Bioch. Biophys., 1979, 19 (3 B), 965-968.

\title{
Adaptation intestinale après entérectomie étendue chez le porcelet en nutrition fractionnée
}

\author{
par J. BATAILLE, Y. REVILLON, B. JEHANNIN, P. DHONT, J. SCHMITZ, \\ C. RICOUR \\ Service de Gastroentérologie pédiatrique ef de Nutrition, \\ Clinique Chirurgicale Infontile et Unité de Recherches de Génétique Médicale, \\ I.N.S.E.R.M., Hôpital des Enfanis malades, 149, rue de Sèvres, \\ 75730, Paris Cedex 15.
}

Summary. Intestinal adaptation after extensive resection in discontinuously fed piglets.

Luminal nutrition is an important factor in the development of intestinal adaptation after resection. However, the effects of continuous or discontinuous feeding are unknown. In this report, we studied their influence in pigs. In the preliminary experiments presented here, the effect of discontinuous feeding ( 2 meals per day) was studied in 12 piglets in which intestinal segments were excised at the duodeno-jejunal flexure and at $90 \mathrm{p} .100$ of the small bowel length at 1 (A), 2 (B) and 3 (C) months of age. 90 p. 100 of the small bowel was resected at 2 months of age (B) in 7 animals (resected group $R$ ); the other 5 served as controls (C). Weight gain was similar in both groups. The excised segments were measured and weighed; protein, disaccharidase, leucine-aminopeptidase and alkaline phosphatase activities were measured in the homogenized mucosa. In both groups the amount of protein per $\mathrm{g}$ of mucosa was about $100 \mathrm{mg}$. In the control group, the amount of protein per $\mathrm{cm}$ and the enzyme activities per $\mathrm{g}$ and per $\mathrm{cm}$ of mucosa did not vary significantly from $A$ to $B$ or from $B$ to $C$. In the resected group, only alkaline phosphatase specific activity varied significantly. On the contrary, protein and activities per $\mathrm{cm}$ increased moderately and more in the jejunum than in the ileum from $A$ to $B$; they rose very significantly from $B$ to $C$ and as compared to controls. This elevation was most pronounced in the ileum. This study confirmed that adaptation of the digestive capacity after extensive resection in pigs 1) occurs through an increase of the mucosal protein mass and of all brush border activities per $\mathrm{cm}$, suggesting mucosal hyperplasia, and 2) is more marked in the ileum.

L'adaptation de l'intestin après résection dépend au moins en partie de la présence de nutriments dans la lumière (Feldman ef al., 1976). L'influence de leur mode d'administration - continu ou fractionné - est toutefois ignorée, ce qui nous a incité à l'étudier chez le porc. Dans un premier temps, qui constitue le sujet de ce travail, a été analysé l'effet de la nutrition fractionnée.

\section{Matériel et méthodes.}

Douze porcelets âgés d'environ un mois et pesant 10,3 $\mp 1,3 \mathrm{~kg}(\mathrm{~m} \mp 1 \sigma)$ nourris en 2 repas/jour avec du Realmentyl (R), ont subi 3 séries de 2 prélèvements chirurgi- 
caux à l'angle de Treitz (J) et à 90 p. 100 de la longueur initiale de l'intestin (I) à 1 mois (A), 2 mois (B) et 3 mois (C); 7 ont subi en outre en $B$ une résection de 90 p. 100 de l'intestin grêle à partir de l'angle de Treitz et constituent le groupe réséqué (R), les 5 autres formant le groupe témoin ( $T$, fig.). La prise de poids a été semblable dans les 2 groupes (en $C, T=25,0 \mathrm{~kg}, R=20,5 \mathrm{~kg}$, différence non significative). Les segments prélevés ont été mesurés et pesés ; la muqueuse a été homogénéisée dans un tampon $0,002 \mathrm{M}$ Tris-0,05 M Mannitol, pH 7,2. Les protéines ef les activités saccharasique (S), maltasique (M), glucamylasique (GA), lactasique (L), de la leucine-aminopeptidase (LAP) et phosphatasique alcaline (PA) ont été dosées selon des méthodes déjà décrites (Schmitz ef al., 1973).

\section{Protocole experimental}

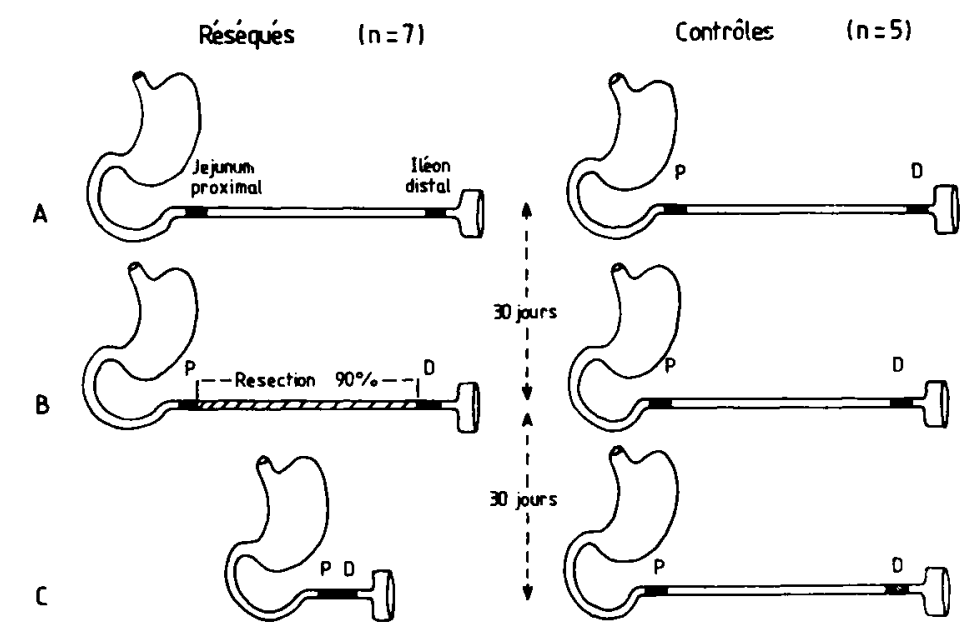

FIG. - Protocole experimental. Douze porcelets (7 réséqués et 5 contrôles) ont subi 2 prélèvements chirurgicaux d'environ $5 \mathrm{~cm}$ à l'angle de Treitz (Jejunum proximal) et à $90 \mathrm{p} .100$ de la longueur de l'intestin (lleon distal) à l'âge de 1 mois puis de 2 mois, les réséqués subissant alors en outre l'ablation de 90 p. 100 de l'intestin grêle à partir de l'angle de Treitz ; à 3 mois tous les animaux ont été sacrifiés après un troisième prélèvement proximal et distal. Durant toute l'expérimentation les porcelets ont été nourris 2 fois par jour avec du Realmentyl $\left({ }^{R}\right)$.

\section{Résultats.}

Dans les 2 groupes le taux de protéines par g de muqueuse reste voisin de $100 \mathrm{mg}$. Dans le groupe témoin les variations du taux de protéines par $\mathrm{cm}$ et des activités enzymatiques par $g$ et par $\mathrm{cm}$ de $A$ en $B$ et de $B$ en $C$ ne sont pas significatives. Les résultats (tabl.) concernant le groupe réséqué peuvent être analysés de la façon suivante : durant la première partie de l'étude, de $A$ en $B$, avant la résection, les activités spécifiques (UI/g protéine de muqueuse) ne varient significativement ni dans le jéjunum, ni dans l'iléon, sauf pour la GA et la $M$ dans le jéjunum. Par contre, durant le même temps, la quantité de protéines de muqueuse et les activités enzymatiques par $\mathrm{cm}$ doublent dans l'ensemble au niveau du jéjunum, à l'exception de celle de la lactase alors que dans l'iléon elles ne sont multipliées que par 1,3 en moyenne. Après la résec- 
tion de $B$ en $C$, les activités spécifiques sauf celle de la $P A, n$ 'augmentent là encore ni dans le jéjunum, ni dans l'iléon. Au contraire, exprimées par cm, toutes sauf la lactase, sont multipliées par 2 dans le jéjunum et par 3 ou 4 dans l'iléon, ces variations étant parallèles à celles des protéines par $\mathrm{cm}$. De plus la comparaison des activités par $\mathrm{cm}$ dans l'iléon mais non dans le jéjunum, en $C$, entre les réséqués et les fémoins est toujours hautement significative ( $P<0,001$, sauf pour la $G A$ ef la LAP où $p<0,01$ ). II faut cependant noter que cet accroissement moyen ne reflète pas la grande variabilité de son amplitude (de 1 à 3 ou 4) d'un animal à l'autre, quelle que soit sa croissance pondérale.

\section{TABLEAU}

Protéines ef activités enzymatiques chez les porcs réséqués.

(Rapport d'un temps au temps précédent)

\begin{tabular}{|c|c|c|c|c|c|c|c|c|c|c|c|c|c|c|}
\hline & \multicolumn{2}{|c|}{ Protéines } & \multicolumn{2}{|c|}{ S } & \multicolumn{2}{|c|}{ GA } & \multicolumn{2}{|c|}{$M$} & \multicolumn{2}{|c|}{$\mathbf{L}$} & \multicolumn{2}{|c|}{ LAP } & \multicolumn{2}{|c|}{ PA } \\
\hline & $B / A$ & C/B & B/A & $\mathrm{C} / \mathrm{B}$ & $B / A$ & $\mathrm{C} / \mathrm{B}$ & $B / A$ & $C / B$ & $B / A$ & $\mathrm{C} / \mathrm{B}$ & $B / A$ & $C / B$ & B/A & $C / B$ \\
\hline $\int\{\operatorname{par} g(1) \ldots$ & 1,0 & 1,0 & 1,3 & 1,3 & $\begin{array}{c}1,5 \\
*\end{array}$ & 1,1 & $\stackrel{1,4}{*}$ & 1,1 & 0,8 & 0,6 & 1,2 & 1,3 & 1,3 & $\begin{array}{c}1,6 \\
*\end{array}$ \\
\hline par cm ... & $\begin{array}{l}2,0 \\
* * *\end{array}$ & $\begin{array}{c}1,4 \\
*\end{array}$ & 2,0 & $\begin{array}{c}2,0 \\
*\end{array}$ & 2,4 & $\begin{array}{l}1,9 \\
*\end{array}$ & 2,3 & 1,7 & 1,4 & 0,9 & $\begin{array}{l}1,9 \\
* *\end{array}$ & $\begin{array}{l}1,9 \\
* *\end{array}$ & 1,9 & $\begin{array}{l}2,8 \\
* * *\end{array}$ \\
\hline I $\{$ par $g(1) \ldots$ & 1,0 & 1,1 & 1,0 & 1,2 & 1,1 & 1,9 & 1,2 & 1,5 & 0,8 & 1,1 & 1,0 & $\begin{array}{c}1,6 \\
*\end{array}$ & 0,9 & $\begin{array}{l}1,6 \\
* * *\end{array}$ \\
\hline par $\mathrm{cm}$ & 1,3 & $\begin{array}{r}2,4 \\
* * * *\end{array}$ & 1,3 & $\begin{array}{c}3,1 \\
* * * *\end{array}$ & $\stackrel{1,5}{*}$ & $\begin{array}{r}4,0 \\
* * * *\end{array}$ & $\begin{array}{c}1,6 \\
*\end{array}$ & $\begin{array}{c}3,1 \\
* * * *\end{array}$ & 1,0 & $\begin{array}{r}2,0 \\
* * * *\end{array}$ & 1,4 & $\begin{array}{l}3,9 \\
* * *\end{array}$ & 1,4 & $\begin{array}{r}4,2 \\
* * * *\end{array}$ \\
\hline
\end{tabular}

(1) $\mathrm{g}$ de muqueuse fraîche pour les protéines, g de protéines pour les activités enzymatiques. * $\mathrm{P}<0,05 ;{ }^{* *} \mathrm{P}<0,02 ; * * * \mathrm{p}<0,01 ;{ }^{* * * *} \mathrm{P}<0,001$.

\section{Discussion.}

Cetfe étude montre, comme le seul travail récent (Aumaitre et Corring, 1978) concernant la maturation de l'intestin du porcelet après 1 mois, que celle-ci continue plus activement entre un et deux mois de vie dans le jéjunum que dans l'iléon et est due surtout à cet âge à une augmentation de la masse protéique de la muqueuse, et non plus, sauf pour l'activité maltasique, comme au cours de la période néonatale, à une augmentation des activités spécifiques; le faił que l'activité glucamylasique varie parallèlement à l'activité maltasique laisse entendre d'autre part que chez le porc, à cet âge, la maturation de cette dernière est surtout le fait de la glucamylase. De même, les résultats présentés confirment les études antérieures en montrant que l'adaptation après résection sublotale s'effectue sans augmentation (McCarthy et Kim, 1973) des activités spécifiques, sans leur diminution (Weser et Hernandez, 1971) non plus, du fait sans doute du jeune âge des onimaux, mais par un accroissement de la masse de muqueuse par cm (McCarthy et Kim, 1973 ; Weser et Hernandez, 1971) et qu'elle est plus importante dans l'iléon que dans le jéjunum (Dowling et Booth, 1967 ; Margolis et al., 1977). Toutes les activités digestives mesurées, y compris celles de la GA et de la 
LAP jusqu'à présent non étudiées, variant de façon parallèle, il est possible d'attribuer avec vraisemblance cette variation à l'augmentation du nombre des cellules absorbantes (Weser et Hernandez, 1971). Il est remarquable que le même type de modification tissulaire soit en cause au cours des phases tordives de la maturation de l'intestin et lors de son adaptation après résection ; les mêmes facteurs tropiques (hormonaux ?) (Weser, 1978) pourraient donc être en jeu dans les deux cas. L'influence éventuelle du mode d'administration de l'alimentation sur ceux-ci reste à démontrer dans un travail à venir concernant des porcelets nourris à débit constant pendant le nycthemère.

Commission CNERNA Digestion-Absorption/Association des Physiologistes,

Paris 5-6 octobre 1978.

\section{Références}

AUMAITRE A., CORRING T., 1978. Development of digestive enzymes in the piglet from birth to 8 weeks. Il. Intestine and intestinal disaccharidases. Nutr. Metab., 22, 244-255.

DOWLING R. H., BOOTH C. C., 1967. Structural and functional changes following small intestinal resection in the rat. Clin. Sci., 32, 139-149.

FELDMAN E. J., DOWLING R. H., MCNAUGHTON J., PETERS T. J., 1976. Effects of oral versus intravenous nutrition on intestinal adaptation after small bowel resection in the dog. Gastroenterology, 70, 712-719.

MARGOLIS A., RICOUR C., HAROUCHI A., GUYOT M., LAOUARI D., BALSAN S., 1977. Intestinal calcium-binding protein 3 months after massive small bowel resection in the piglet. Amer. J. clin. Nutr., 30, 2041-2046.

MCCARTHY D. M., KIM Y. S., 1973. Changes in sucrase, enterokinase, and peptide hydrolase after intestinal resection. J. clin. Invest., 52, 942-951.

SCHMITZ J., PREISER H., MAESTRACCI D., GHOSH B. K., CERDA J. J., CRANE R. K., 1973. Purification of the human intestinal brush border membrane. Biochim. biophys. Acta, 323, 98-112.

WESER E., HERNANDEZ M. H., 1971. Studies of small bowel adaptation after intestinal resection in the rat. Gastroenterology, 60, 69-75.

WESER E., 1978. Role of gastrin in intestinal adaptation after small bowel resection. Gastroenterology, 75, 323-324. 